\title{
Propagation of Sound Waves in the Atmosphere
}

\author{
B. GUTENBERG \\ California Institute of Technology, Pasadena, California
}

(Received August 31, 1942)

\begin{abstract}
The effect of humidity on the velocity of sound waves is investigated (Eq. (5), Table I). The radius of curvature for a ray of sound which is propagated in the direction of the wind is given [Eq. (13)] and discussed. The amplitudes of sound waves as a function of the distance are given [Eq. (17)], and the relative importance of the quantities involved is discussed.
\end{abstract}

\section{INTRODUCTION}

$T$ HE problem of the propagation of sound waves in the atmosphere, though of practical interest, seems to have been somewhat neglected. The following article is a contribution to certain aspects of this problem.

\section{SOUND VELOCITY IN HUMID AIR}

It is usually stated in publications that the effect of humidity on the sound velocity is small. Assuming a purely adiabatic process, the sound velocity $V$ in a gas is given by

$$
V=(p \kappa / \rho)^{\frac{1}{2}}=(R T \kappa)^{\frac{1}{2}},
$$

where $p=$ pressure, $\kappa=C_{p} / C_{v}$ is the ratio of the specific heats at constant pressure and at constant volume, $\rho=$ density, $R=$ gas constant for the given gas, and $T=$ absolute temperature. For dry air $R=2.87 \times 10^{6} \mathrm{~cm}^{2} / \mathrm{sec}^{2}$ and $\kappa=1.4$ which gives

$$
V=20.05 \sqrt{ } T(\mathrm{~m} / \mathrm{sec} .) \text {. }
$$

For $T=273^{\circ} \mathrm{K}$, Eq. (2) gives $V=331.6 \mathrm{~m} / \mathrm{sec}$. which is very close to the observed value $331.45 .{ }^{1}$ For all temperatures to be expected in the atmosphere, the values calculated from (2) agree with the observations within the limits of error involved in the problems of this paper.

In humid air, $\rho, R$, and $\kappa$ depend on the partial pressure $h$ (sometimes called tension) of the water vapor. Using the index $a$ for dry air, $w$ for water vapor, and $h$ for humid air, we find from (1)

$$
\begin{aligned}
V_{h}=V_{a}\left(\frac{\kappa_{h}}{\kappa_{a}} \frac{R_{h}}{R_{a}}\right)^{\frac{1}{2}}=V_{a} & \left(\frac{\kappa_{h}}{\kappa_{a}} \frac{p}{p-\left(1-\frac{\rho_{w}}{\rho_{a}}\right) h}\right)^{\frac{1}{2}} \\
& =V_{a}\left(\frac{\kappa_{h}}{\kappa_{a}} \frac{p}{p-0.38 h}\right)^{\frac{1}{2}} .
\end{aligned}
$$

${ }^{1}$ H. C. Hardy, D. Telfair, and W. H. Pielemeier, "The velocity of sound in air," J. Acous. Soc. Am. 13, 226 (1942).
With sufficient approximation ${ }^{2}$

$$
\frac{\kappa_{h}}{\kappa_{a}}=\frac{(p-h)\left(\kappa_{w}-1\right)+h \frac{\kappa_{w}}{\kappa_{a}}\left(\kappa_{a}-1\right)}{(p-h)\left(\kappa_{w}-1\right)+h\left(\kappa_{a}-1\right)}=1-0.1 \frac{h}{p-h},
$$

using $\kappa_{w}=1.3$ and $\kappa_{a}=1.4$. Introducing this result in (3) we find approximately

$$
V_{h}=V_{a}+\stackrel{h}{0.14-V_{a}}=V_{a}+H, \quad H=\underset{p}{h} \stackrel{h}{p} V_{a} .
$$

The correction $H$ depends on the ratio of the partial pressure $h$ of the water vapor to the pressure $p$ (elevation above the ground). Near the ground, we may take as averages $p=760 \mathrm{~mm}$ $\mathrm{Hg}, V=340 \mathrm{~m} / \mathrm{sec}$. and find with these figures approximately

$$
H_{0}=0.063 h(H \text { in } \mathrm{m} / \mathrm{sec} . h \text { in } \mathrm{mm} \mathrm{Hg}) .
$$

In Table I, calculated values for the approximate correction $H_{0}$ near the ground are given. In air with a relative humidity of $x$ percent, the corrections must be multiplied by $x / 100$. In warm, humid air, the corrections may exceed 1 percent of the value for dry air.

\section{EFFECT OF WIND ON THE PATH OF SOUND WAVES}

The equations for the paths of sound waves

\begin{tabular}{|c|c|c|c|c|c|c|c|c|c|c|}
\hline$h$ & $\begin{array}{r}+50 \\
92.5 \\
5.8\end{array}$ & $\begin{array}{r}45 \\
71.9 \\
4.5\end{array}$ & $\begin{array}{r}40 \\
55.3 \\
3.5\end{array}$ & $\begin{array}{r}35 \\
42.2 \\
2.7\end{array}$ & $\begin{array}{r}30 \\
31.8 \\
2.0\end{array}$ & $\begin{array}{r}25 \\
23.8 \\
1.5\end{array}$ & $\begin{array}{r}20 \\
17.5 \\
1.1\end{array}$ & $\begin{array}{l}10 \\
9.2 \\
0.6\end{array}$ & $\begin{array}{c}0 \\
4.6 \\
0.3\end{array}$ & $\begin{array}{l}-10^{\circ} \mathrm{C} \\
2.1 \mathrm{~mm} \mathrm{Hg} \\
0.1 \mathrm{~m} / \mathrm{sec}\end{array}$ \\
\hline
\end{tabular}
in the case that the wind direction forms an angle

TABLE I. Partial pressure $h$ for temperatures $t$ and approximate corrections $H_{0}$ to be added to sound velocity for dry air to find the sound velocity in air of 100 percent humidity near the ground. (Supposed $p=760 \mathrm{~mm} \mathrm{Hg}$, $V=340 \mathrm{~m} / \mathrm{sec}$. or $T=287^{\circ} \mathrm{K}$.)

${ }^{2}$ E. Luebcke, "Schallgeschwindigkeit," Handbuch der Physik 8, 626 (Śpringer, Berlin, 1927). 


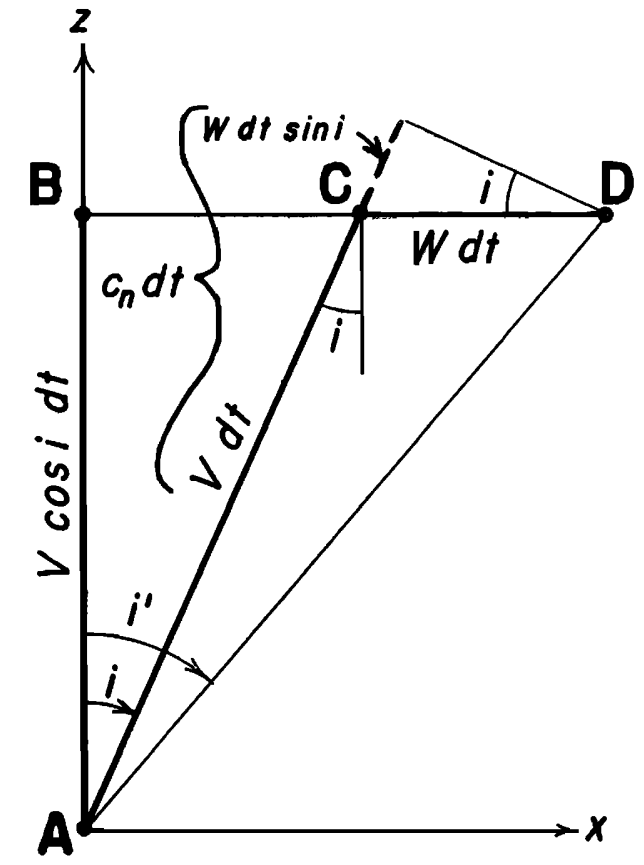

FIG. 1.

$\varphi$ with the horizontal component of the sound path are too complicated ${ }^{3,4}$ to be useful, even when it is assumed that the wind has no vertical component and that its direction does not change with height. Usually, the wind component $(W \cos \varphi)$ is taken, and then the equations are applied which hold when the directions of the wind and of the sound waves are in the same vertical plane. However, it seems that even for this simpler case, no equations have been derived ${ }^{5}$ which in general consider terms of the order $W / V$ ( $W=$ wind velocity, $V=$ sound velocity in still air). Complications arise mainly from the fact that the wave front connecting points with equal times of arrival of the waves is no longer perpendicular to wave paths. The sound velocity component normal to the wave front is $c_{n}=V$ $+W \sin i$ (see Fig. 1). For $c_{n}$ the usual law of refraction holds :

$$
\begin{gathered}
c_{n} / \sin i=\text { constant }=C=V / \sin i+W, \\
\sin i=V /(C-W) .
\end{gathered}
$$

From Fig. 1:

$\tan i^{\prime}-\tan i=(B D-B C) / A B=W / V \cos i$,

or approximately $\left(i^{\prime}-i\right)$ in radians $=W \cos i / V$. Thus, in applying Eq. (8) to $i^{\prime}$ instead of to $i$, an error of the order $W / V$ is introduced, unless $i$ is close to $90^{\circ}$.

The ray equation follows from Fig. 1:

$x^{\prime}=d x / d z=B D / A B=(V \sin i+W) / V \cos i$.

The absolute value of the radius of curvature $r$ is given by

$$
\begin{aligned}
r=\frac{\left(1+x^{\prime 2}\right)^{\frac{\pi}{2}}}{x^{\prime \prime}}= & \frac{\frac{1}{\cos ^{3} i}\left[1+2 \frac{W}{V} \sin i+\left(\frac{W}{V}\right)^{2}\right]^{\frac{3}{2}} V^{2} \cos ^{2} i}{V^{2} \frac{d i}{d z}+V \cos i \frac{d W}{d z}-W \cos i \frac{d V}{d z}+W V \sin i \frac{d i}{d z}} \\
= & \frac{V\left[1+2 \frac{W}{V} \sin i+\left(\frac{W}{V}\right)^{2}\right]^{\frac{3}{2}}}{\frac{\cos i}{V}\left[\left(V^{2}+W V \sin i\right) \frac{d i}{d z}+\cos i\left(V \frac{d W}{d z}-W \frac{d V}{d z}\right)\right]}
\end{aligned}
$$

Introducing from Eq. (8)

$$
\cos i \frac{d i}{d z}=\frac{(C-W) \frac{d V}{d z}+V \frac{d W}{d z}}{(C-W)^{2}}=\left(\frac{d V}{d z}+\frac{d W}{d z} \sin i\right) \frac{\sin i}{V}
$$

3 R. Emden, "Beiträge zur Thermodynamik der Atmosphäre," Meteorol. Zeits. 35, 14, 74, and 114 (1918).

4 B. Gutenberg, "Die Schallausbreitung in der Atmosphäre," Handbuch der Geophysik 9, 89-144 (Gebr. Borntraeger, Berlin, 1932). P. Duckert, "Ueber die Ausbreitung von Explosionswellen in der Erdatmosphäre," Ergeb. d. Kosm. Physik 1, 236-290 (1931). With 193 references.

${ }_{5}^{5}$ B. Haurwitz, "The propagation of sound through the atmosphere," J. Aer. Sci. 9, 35 (1941). 
gives

$$
r=\frac{V\left[1+2 \frac{W}{V} \sin i+\left(\frac{W}{V}\right)^{2}\right]^{\frac{3}{2}}}{\frac{d V}{d z}\left(\sin i-\frac{W}{V} \cos 2 i\right)+\frac{d W}{d z}\left(1+\frac{W}{V} \sin ^{3} i\right)} .
$$

The rays are concave towards the earth if the denominator is positive. Introducing the absolute temperature $T$ from (2),

$$
\begin{gathered}
d V / d z=(10 / \sqrt{ } T)(d T / d z), \\
r=\frac{V\left[1+2 \frac{W}{V} \sin i+\left(\frac{W}{V}\right)^{2}\right]^{\frac{3}{2}}}{\frac{10}{\sqrt{ } T}\left(\sin i-\frac{W}{V} \cos 2 i\right) \frac{d T}{d z}+\left(1+\frac{W}{V} \sin ^{3} i\right) \frac{d W}{d z}} .
\end{gathered}
$$

$r=$ radius of curvature, $T=$ absolute temperature, $V=20 \sqrt{ } T$ (sound velocity in still air), $W=$ wind velocity, $i=$ angle of incidence, $z=$ elevation. For practical purposes, the numerator in (13) may be written :

$$
V\left[1+\frac{W}{V} \sin i\right]^{3} \text { or }[V+3 W \sin i]
$$

Usually $d T / d z$ is negative. Writing $d T / d z=-\gamma$, $d W / d z=a$, the condition for a curvature of the rays concave toward the ground is given by

$$
a\left(1+\frac{W}{V} \sin ^{3} i\right)>\frac{10 \gamma}{\sqrt{ } T}\left(\sin i-\frac{W}{V} \cos 2 i\right)
$$

For very flat rays $\left(i\right.$ near $\left.90^{\circ}\right)$, this leads to $a>10 \gamma / \sqrt{ } T$; for example, for $T=289^{\circ}: a>0.59 \gamma$, regardless of the elevation $z$. If the decrease of temperature (lapse rate $\gamma$ ) is about $6^{\circ}$ per $\mathrm{km}$, the wind must increase by more than $3 \frac{1}{2} \mathrm{~m} / \mathrm{sec}$. per $\mathrm{km}$ near the highest point of the ray to produce a return of the sound to earth. A wind increase of this order of magnitude had already been used in the early literature. It also has been pointed out that for our problem the changes in $T$ and $W$ with height are much more important than the absolute values of both quantities.

For $i=0$, (13) gives approximately

$$
r=V /(a+W \gamma / 2 T)
$$

If the temperature decreases with elevation, a straight ray directed vertically upward is possible only if the wind decreases with elevation in such a way that the denominator in (15) is zero. Usually this decrease need not exceed materially 1 percent of the wind velocity per kilometer.

\section{AMPLITUDES OF SOUND WAVES THROUGH THE ATMOSPHERE}

We suppose that there is no wind, and that sound waves produced at $A$ in Fig. 2 start with the same energy in all directions within the small half-sphere. The energy between two cones formed by rays with angles of incidence $i$ and $i+\epsilon$, respectively, is given by

$$
a[\cos i-\cos (i+\epsilon)],
$$

where $a$ depends on the energy at the source and the radius of the sphere. The absorption on the path $D$ (Fig. 3) is given to a first approximation

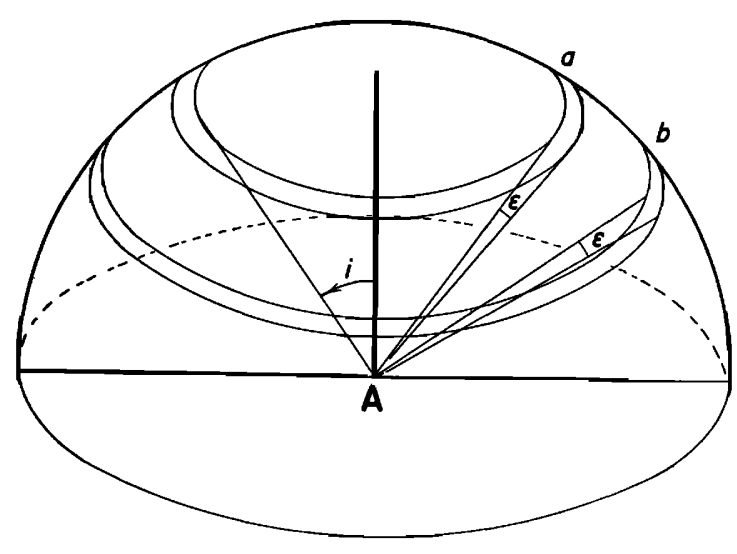

FIG. 2. 


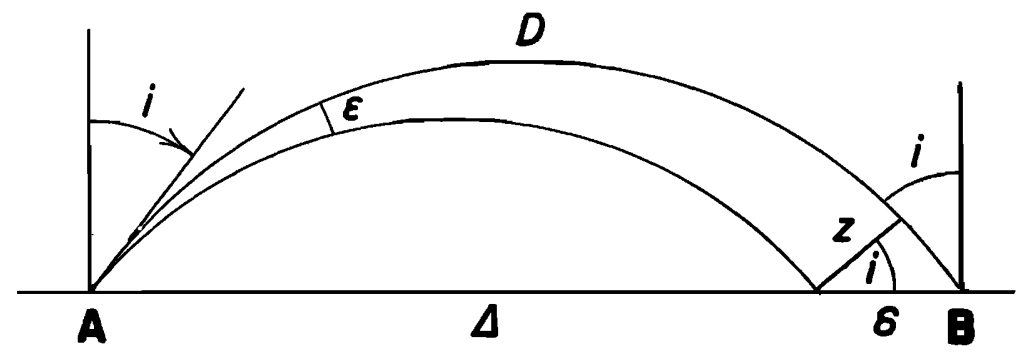

FIG. 3.

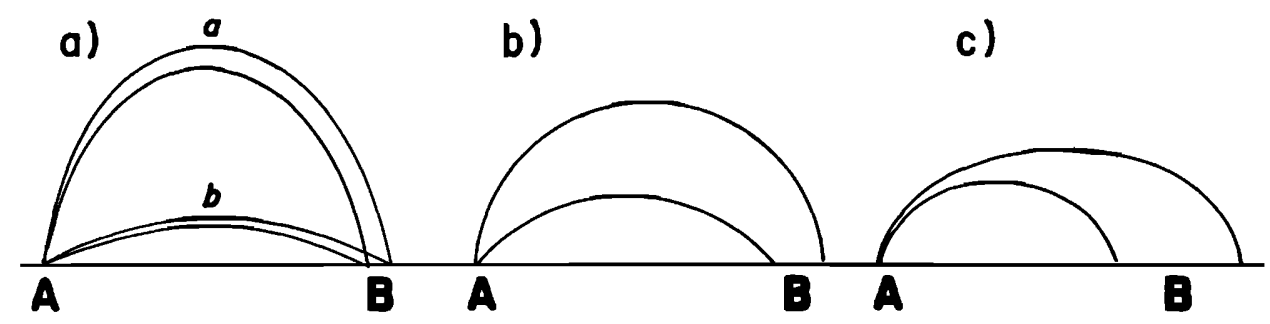

FIG. 4.

by $e^{-k D}$. The remaining energy arrives on a zone of area $Z$ perpendicular to the rays:

$Z=\pi\left[\Delta^{2}-(\Delta-\delta)^{2}\right] \cos i$

= approximately $2 \pi \Delta \delta \cos i$.

Consequently, the energy flux at the distance $\Delta$ is given by

$$
E=\frac{C[\cos i-\cos (i+\epsilon)] e^{-k D}}{\Delta \delta \cos i},
$$

where $C$ is a constant, depending on the original energy. As

$$
\frac{\cos i-\cos (i+\epsilon)}{\delta}=\frac{d \cos i}{d \Delta}=-\sin i \frac{d i}{d \Delta},
$$

$$
E=C e^{-k \nu} \frac{\tan i}{\Delta}\left(-\frac{d i}{d \Delta}\right) .
$$

$E=$ energy flux, $C=$ constant $k=$ absorption coefficient, $D=$ length of sound path, $i=$ angle of incidence, $\Delta=$ distance at the ground.

The factor $\tan i$ is partly a consequence of the fact that near the source less energy passes through $a$ (Fig. 2) than through the larger zone $b$. Under otherwise equal conditions, waves along the path $a$ (Fig. 4a) arrive at $B$ with less energy than those over the path $b$. If $i=90^{\circ}, \delta=\infty$, $d i / d \Delta=0$.

The largest local changes are produced by $d i / d \Delta$. If $i$ changes rapidly with distance (Fig.
$4 \mathrm{~b})$, much energy is concentrated near $B$. On the other hand, in Fig. 4c, it has been assumed that $i$ changes slowly with $\Delta$, the energy per unit near $B$ is small.

To simplify the discussion we assume that the rays are circles (Fig. 5). Then

$$
\begin{gathered}
\cos i=\Delta / 2 r \\
-\sin i(d i / d \Delta)=[r-\Delta(d r / d \Delta)] / 2 r^{2} .
\end{gathered}
$$

Neglecting the absorption factor, we have in this special case

$$
E=\frac{C}{\cos i} \frac{1-\frac{\Delta}{r} \frac{d r}{d \Delta}}{2 r \Delta}=C \frac{1-\frac{\Delta}{r} \frac{d r}{d \Delta}}{\Delta^{2}} .
$$

Equation (18) shows the expected decrease in energy with the square of the distance. The energy at a given point is especially large if the

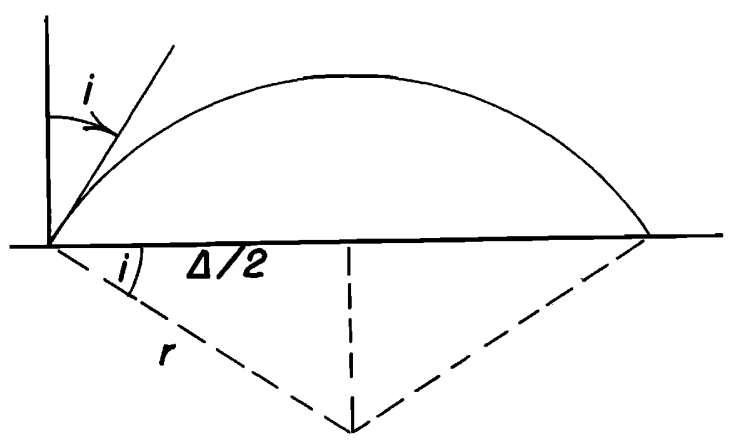

FIG. 5. 
radius of curvature decreases rapidly with distance. According to Eq. (13) this requires that either the wind or the temperature or both increase rapidly with increasing height $z$ in the region of the highest point reached by the sound waves. If this happens, sound waves from gun fire or explosions may be heard at unusually large distances. ${ }^{6}$ On the other hand, the energy decreases considerably when the highest part of the rays enters a region where the radius of curvature increases rapidly.

As long as the rays remain below 20 or $30 \mathrm{~km}$, the absorption factor $e^{-k D}$ plays a smaller role for low tones $(\lambda>10$ meters) than the change in the radius of curvature due to changes of wind and temperature with elevation. Approximately ${ }^{7}$

$$
k=33 l / \lambda^{2},
$$

${ }^{6} \mathrm{~B}$. Gutenberg, "The velocity of sound waves and the temperature in the stratosphere in southern California," Bull. Am. Meteorolog. Soc. 20, 192 (1939). See especially Figs. 1 and 3, pp. 192 and 193.

${ }^{7}$ E. Schrödinger "Zur Akustik der Atmosphäre," Physik. Zeits. 18, 445 (1917). See also J. Kölzer, Meteorol. Zeits. 42, 457 (1925). where $l=$ mean free path of the molecules, $\lambda=$ wave-length. If we assume a constant temperature of $273^{\circ} \mathrm{K}$ in the atmosphere, we find as a first approximation at the height $h$ :

$$
l=l_{0} \frac{p_{0}}{p}=l_{0} e^{h / 8}, \quad k=33 l_{0} \frac{e^{h / 8}}{\lambda^{2}},
$$

where the index zero refers to the ground, and $h$ is measured in $\mathrm{km}$.

Waves corresponding to the lowest audible tones $(\lambda=20 \mathrm{~m})$ suffer an absorption of 1 percent per $\mathrm{km}$ at an elevation of about $60 \mathrm{~km}$. At an elevation of $100 \mathrm{~km}$, only about one-tenth of one percent of the energy of such waves arrives after traversing a distance of one $\mathrm{km}$; for practical purposes the limit of propagation for the lowest audible sound waves over distances of $10 \mathrm{~km}$ or more is about at an elevation of 70 kilometers. From Eq. (20) it follows that for high tones $(\lambda$ in meters) the corresponding elevations decrease roughly by $37 \log (20 / \lambda)$ kilometers. 